\title{
FUSION OF X RAY RADIOGRAPHIC DATA AND ANATOMICAL DATA IN COMPUTED TOMOGRAPHY
}

\author{
Ali Mohammad-Djafari
}

\author{
Laboratoire des Signaux et Systèmes (CNRS-SUPELEC-UPS) \\ École Supérieure d'Électricité \\ Plateau de Moulon, 91192 Gif-sur-Yvette Cedex, France. \\ E_mail: djafarielss.supelec.fr
}

\begin{abstract}
In this paper, we consider an $\mathrm{X}$ ray computed tomography $(\mathrm{CT})$ image reconstruction problem using two different kinds of data: classical X-rays radiographic data and some geometrical informations coming from anatomical atlas and propose new methods based on Bayesian estimation approach for this data fusion problem. We used two kinds of anatomical information: partial knowledge of values in some regions and partial knowledge of the edges of some other regions. We showed the advantages of using such informations on increasing the quality of reconstructions. We also showed some results to analyze the effects of some errors in anatomic data on the reconstructed results.

key words: Bayesian data fusion, Computed tomography, Fusion of radiography and anatomic data.
\end{abstract}

\section{INTRODUCTION}

Data fusion is one of the active area of research in many applications such as non destructive testing (NDT), geophysical imaging, radio-astronomy and medical imaging. We consider the case of this last application with an X ray computed tomography (CT) image reconstruction problem using two different kind of data: classical radiographic (projection) data and some geometrical informations coming from anatomical atlas such as partial knowledge of the anatomic regions and/or the borders of these regions.

The idea of using anatomical data in medical CT imaging is not new. Many works on the subject has been done before. See for example [1, 2, 3, 4, 5, 6]. In [1], the authors proposed methods for using regions borders from anatomical data in medical imaging and the authors in $[2,3,5,6]$ used the knowledge of some of regions themselves. While the application in the first reference concerns medical imaging, the application in the second references concerns industrial non destructive testing. But, combining both regions and borders informations from anatomic data is new. This work is still in development. We give here some preliminary results simulating a fan beam CT problem with more limited number of $\mathrm{X}$ ray data. A few results show comparisons of the results using classical back-projection or filtered back-projection methods with those obtained by the proposed method either using or not the anatomic data. These results show the advantages of using anatomic data when these data are exact and well registered with radiographic data. Some preliminary results show also the sensitivity of the proposed method to some errors in anatomical data due to imperfect registration and other uncertainties.

\section{BAYESIAN APPROACH FOR DATA FUSION}

\subsection{Fusion of homogeneous data}

Assume that we are observing an unknown quantity $\boldsymbol{x}$ through two different measurement systems and obtained two sets of data $\boldsymbol{y}$ and $\boldsymbol{z}$. Assuming that both measurement systems are linear, we have

$$
\left\{\begin{array}{l}
\boldsymbol{y}=\boldsymbol{H}_{1} \boldsymbol{x}+\boldsymbol{\epsilon}_{1} \\
\boldsymbol{z}=\boldsymbol{H}_{2} \boldsymbol{x}+\boldsymbol{\epsilon}_{2}
\end{array}\right.
$$

where $\boldsymbol{H}_{1}$ and $\boldsymbol{H}_{2}$ characterize these systems and $\boldsymbol{\epsilon}_{1}$ and $\boldsymbol{\epsilon}_{2}$ their respective errors.

For example, consider an X ray tomography problem where $\boldsymbol{x}$ represents the mass density of the object and where $\boldsymbol{y}$ and $\boldsymbol{z}$ represent respectively a high resolution projection and a low resolution projection.

We can use directly the Bayesian approach to solve this problem by writing down the posterior law:

$$
p(\boldsymbol{x} \mid \boldsymbol{y}, \boldsymbol{z})=\frac{p(\boldsymbol{y}, \boldsymbol{z} \mid \boldsymbol{x}) p(\boldsymbol{x})}{p(\boldsymbol{y}, \boldsymbol{z})}
$$

Actually, the main difficulty here is to assign $p(\boldsymbol{y}, \boldsymbol{z} \mid \boldsymbol{x})$ and $p(\boldsymbol{x})$. If we assume that the errors associated to the two sets of data are independent then we have $p(\boldsymbol{y}, \boldsymbol{z} \mid \boldsymbol{x})=p(\boldsymbol{y} \mid \boldsymbol{x}) p(\boldsymbol{z} \mid \boldsymbol{x})$, and if we assume to be able to assign $p(\boldsymbol{y} \mid \boldsymbol{x}), p(\boldsymbol{z} \mid \boldsymbol{x})$ and $p(\boldsymbol{x})$. then we can compute the posterior $p(\boldsymbol{x} \mid \boldsymbol{y}, \boldsymbol{z})$. and the calculation can be done more easily. For the purpose of illustration assume the following:

$\boldsymbol{\epsilon}_{1} \sim \mathcal{N}\left(\mathbf{0}, \sigma_{1}^{2} \boldsymbol{I}\right) \rightarrow p\left(\boldsymbol{y} \mid \boldsymbol{x} ; \sigma_{1}^{2}\right) \propto \exp \left[-\frac{1}{2 \sigma_{1}^{2}}\left|\boldsymbol{y}-\boldsymbol{H}_{1} \boldsymbol{x}\right|^{2}\right]$
$\boldsymbol{\epsilon}_{2} \sim \mathcal{N}\left(\mathbf{0}, \sigma_{2}^{2} \boldsymbol{I}\right) \rightarrow p\left(\boldsymbol{z} \mid \boldsymbol{x} ; \sigma_{2}^{2}\right) \propto \exp \left[-\frac{1}{2 \sigma_{2}^{2}}\left|\boldsymbol{z}-\boldsymbol{H}_{2} \boldsymbol{x}\right|^{2}\right]$
$\boldsymbol{x} \sim \mathcal{N}(\boldsymbol{m}, \Sigma) \rightarrow p(\boldsymbol{x} \mid \boldsymbol{m}, \boldsymbol{\Sigma}) \propto \exp \left[-\frac{1}{2}[\boldsymbol{x}-\boldsymbol{m}]^{t} \boldsymbol{\Sigma}^{-1}[\boldsymbol{x}-\boldsymbol{m}]\right]$

Indeed, assume that the hyper-parameters $\left(\sigma_{1}^{2}, \sigma_{2}^{2}, \boldsymbol{m}, \boldsymbol{\Sigma}\right)$ are given. Then we can use, for example, the MAP estimate, given by:

$$
\begin{aligned}
\widehat{\boldsymbol{x}} & =\arg \max \boldsymbol{x}\{p(\boldsymbol{x} \mid \boldsymbol{y}, \boldsymbol{z})\} \\
& =\arg \min \boldsymbol{x}\left\{J(\boldsymbol{x})=J_{1}(\boldsymbol{x})+J_{2}(\boldsymbol{x})+J_{3}(\boldsymbol{x})\right\}
\end{aligned}
$$

with

$J_{1}(\boldsymbol{x})=\frac{1}{2 \sigma_{1}^{2}}\left\|\boldsymbol{y}-\boldsymbol{H}_{1} \boldsymbol{x}\right\|^{2}, \quad J_{2}(\boldsymbol{x})=\frac{1}{2 \sigma_{2}^{2}}\left\|\boldsymbol{z}-\boldsymbol{H}_{2} \boldsymbol{x}\right\|^{2}$ and

$J_{3}(\boldsymbol{x})=\frac{1}{2}[\boldsymbol{x}-\boldsymbol{m}]^{t} \boldsymbol{\Sigma}^{-1}[\boldsymbol{x}-\boldsymbol{m}]$.

However, in practical applications, the data come from different processes and it is more difficult to relate the two sets of data directly to the same unknown quantity.

\subsection{Fusion of non homogeneous data}

Consider a more realistic data fusion problem, where we have two different kinds of data. As an example assume a tomographic image reconstruction problem where we have a set of data $\boldsymbol{y}$ obtained by an $\mathrm{X}$ ray and a set of data $\boldsymbol{z}$ obtained by an ultrasound probing system. The $\mathrm{X}$ ray data are related to the mass density $\boldsymbol{x}$ of the matter while the ultrasound data are related to the acoustic reflectivity $\boldsymbol{r}$ of the matter which is more related to the changes of material mass density inside the object and gives more information on the borders of different homogeneous regions. 
One approach proposed and used by the author [7] and by other collaborators Gautier et al. [8, 6, 9] is based on a compound Markovian model where the body object $\boldsymbol{o}$ is assumed to be composed of three related quantities:

$$
\boldsymbol{o}=\{\boldsymbol{r}, \boldsymbol{x}\}=\{\boldsymbol{q}, \boldsymbol{a}, \boldsymbol{x}\}
$$

where $\boldsymbol{q}$ is a binary vector representing the positions of the discontinuities (edges) in the body, $\boldsymbol{a}$ a vector containing the reflectivity values such that when $q_{j}=0$ then $r_{j}=0$ and when $q_{j}=1$ then $r_{j}=a_{j}$ and $a_{j}$ must be related in some way to changes of density, i.e. $g\left(x_{j+1}-x_{j}\right)$, where $g$ can be any monotonic increasing function. But, in practice, it is not easy to account for this dependence.

With this model we can write

$$
p(\boldsymbol{o})=p(\boldsymbol{x}, \boldsymbol{a}, \boldsymbol{q})=p(\boldsymbol{x} \mid \boldsymbol{a}, \boldsymbol{q}) p(\boldsymbol{a} \mid \boldsymbol{q}) p(\boldsymbol{q})
$$

and using the Bayes rule, we have

$$
\begin{aligned}
p(\boldsymbol{x}, \boldsymbol{a}, \boldsymbol{q} \mid \boldsymbol{y}, \boldsymbol{z}) \propto & p(\boldsymbol{y}, \boldsymbol{z} \mid \boldsymbol{x}, \boldsymbol{a}, \boldsymbol{q}) p(\boldsymbol{x}, \boldsymbol{a}, \boldsymbol{q}) \\
& =p(\boldsymbol{y}, \boldsymbol{z} \mid \boldsymbol{x}, \boldsymbol{a}, \boldsymbol{q}) p(\boldsymbol{x} \mid \boldsymbol{a}, \boldsymbol{q}) p(\boldsymbol{a} \mid \boldsymbol{q}) p(\boldsymbol{q})
\end{aligned}
$$

We illustrate this approach by making the following assumptions: - Conditional independence of $\boldsymbol{y}$ and $\boldsymbol{z}$ :

$$
p(\boldsymbol{y}, \boldsymbol{z} \mid \boldsymbol{x}, \boldsymbol{a}, \boldsymbol{q})=p(\boldsymbol{y} \mid \boldsymbol{x}) p(\boldsymbol{z} \mid \boldsymbol{a}, \boldsymbol{q})=p(\boldsymbol{y} \mid \boldsymbol{x}) p(\boldsymbol{z} \mid \boldsymbol{r}) .
$$

- Gaussian laws for $\epsilon_{1}$ and $\epsilon_{2}$ which results to:

$$
\begin{array}{ll}
p\left(\boldsymbol{y} \mid \boldsymbol{x} ; \sigma_{1}^{2}\right) \propto & \exp \left[-\frac{1}{2 \sigma_{1}^{2}}\left\|\boldsymbol{y}-\boldsymbol{H}_{1} \boldsymbol{x}\right\|^{2}\right] \\
p\left(\boldsymbol{z} \mid \boldsymbol{r} ; \sigma_{2}^{2}\right) \propto & \exp \left[-\frac{1}{2 \sigma_{2}^{2}}\left\|\boldsymbol{z}-\boldsymbol{H}_{2} \boldsymbol{r}\right\|^{2}\right]
\end{array}
$$

- Bernoulli law for $\boldsymbol{q}: \quad p(\boldsymbol{q}) \propto \sum_{i=1}^{n} q_{i}^{\lambda}\left(1-q_{i}\right)^{1-\lambda}$

- Gaussian law for $\boldsymbol{r} \mid \boldsymbol{q}$ or equivalently for $\boldsymbol{a} \mid \boldsymbol{q}$ :

$$
p(\boldsymbol{a} \mid \boldsymbol{q}) \propto \exp \left[-\frac{1}{2 \sigma_{a}^{2}} \boldsymbol{a}^{t} \boldsymbol{Q} \boldsymbol{a}\right], \quad \boldsymbol{Q}=\operatorname{diag}\left[q_{1}, \ldots, q_{n}\right]
$$

- Independence of $\boldsymbol{x}$ and $\boldsymbol{a}$ and a Markovian model for $\boldsymbol{x} \mid \boldsymbol{q}$ :

$$
p(\boldsymbol{x} \mid \boldsymbol{a}, \boldsymbol{q})=p(\boldsymbol{x} \mid \boldsymbol{q}) \propto \exp [-U(\boldsymbol{x} \mid \boldsymbol{q})]
$$

Then, based on

$$
p(\boldsymbol{x}, \boldsymbol{a}, \boldsymbol{q} \mid \boldsymbol{y}, \boldsymbol{z}) \propto p(\boldsymbol{y} \mid \boldsymbol{x}) p(\boldsymbol{z} \mid \boldsymbol{a}) p(\boldsymbol{x} \mid \boldsymbol{a}, \boldsymbol{q}) p(\boldsymbol{a} \mid \boldsymbol{q}) p(\boldsymbol{q})
$$

we proposed the following schemes to estimate either $\boldsymbol{x}$ or both $(\boldsymbol{x}, \boldsymbol{r})$ or equivalently $(\boldsymbol{x}, \boldsymbol{a}, \boldsymbol{q})$ :

- Simultaneous estimation of all the unknowns with the joint MAP estimation (JMAP):

$$
(\widehat{\boldsymbol{x}}, \widehat{\boldsymbol{a}}, \widehat{\boldsymbol{q}})=\arg \max _{(\boldsymbol{x}, \boldsymbol{a}, \boldsymbol{q})}\{p(\boldsymbol{x}, \boldsymbol{a}, \boldsymbol{q} \mid \boldsymbol{y}, \boldsymbol{z})\}
$$

- First estimate $\boldsymbol{q}$ and $\boldsymbol{a}$ using only $\boldsymbol{z}$ and then use them to estimate $\boldsymbol{x}$ :

$$
\left\{\begin{array}{l}
(\widehat{\boldsymbol{q}}, \widehat{\boldsymbol{a}})=\arg \max _{\boldsymbol{q}, \boldsymbol{a}}\{p(\boldsymbol{q}, \boldsymbol{a} \mid \boldsymbol{z})\} \\
\widehat{\boldsymbol{x}}=\arg \max _{\boldsymbol{x}}\{p(\boldsymbol{x} \mid \boldsymbol{y}, \widehat{\boldsymbol{a}}, \widehat{\boldsymbol{q}})\}
\end{array}\right.
$$

- First estimate only $\boldsymbol{q}$ using $\boldsymbol{z}$ and then estimate $\boldsymbol{x}$ using $\widehat{\boldsymbol{q}}$ and the data $\boldsymbol{y}$ :

$$
\left\{\begin{array}{l}
\widehat{\boldsymbol{q}}=\arg \max _{\boldsymbol{q}}\{p(\boldsymbol{q} \mid \boldsymbol{z})\} \\
\widehat{\boldsymbol{x}}=\arg \max _{\boldsymbol{x}}\{p(\boldsymbol{x} \mid \boldsymbol{y}, \widehat{\boldsymbol{q}})\}
\end{array}\right.
$$

Other schemes are possible [7]. In all these schemes, the detection steps (estimation of $\boldsymbol{q}$ ) is very difficult and computationally demanding due to the need of marginalization and a combinatorial optimization algorithm. One way to take over this difficulty is to model the object only by $(\boldsymbol{r}, \boldsymbol{x})$ without decomposing $\boldsymbol{r}$ in $(\boldsymbol{q}, \boldsymbol{a})$ anymore. However, we must catch the information on $\boldsymbol{r}$ which is almost always equal to zero (in homogeneous regions) and can take any real value in the borders of these regions. This can be down through a better choice for $p(\boldsymbol{r})$. For example, a generalized Gaussian law for $p(\boldsymbol{r})$ :

$$
p(\boldsymbol{r}) \propto \exp \left[-\alpha \sum_{j}\left|r_{j}\right|^{\beta}\right] \quad \text { with } 1 \leq \beta \leq 2 .
$$

(in place of Gaussian law which is the special case for $\beta=2$ ), can be used to translate this concentration around zero while giving the possibility to have large values, thanks to long-tailed character of this distribution for the values of $\beta$ near to one.

Based on this remark, a more realistic solutions has been proposed in previous works $[10,9,7,11]$ which is described briefly.

\subsection{Proposed method}

Use the ultrasound data $\boldsymbol{z}$ to detect the locations of some of the boundaries and use $\mathrm{X}$ ray data to make an intensity image preserving the positions of these discontinuities:

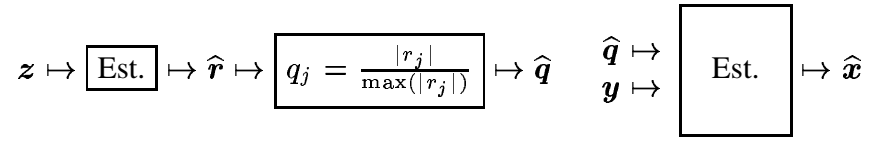

For the first part, with the assumptions made, we have

with

$$
\begin{gathered}
\widehat{\boldsymbol{r}}=\arg \max _{\boldsymbol{r}}\{p(\boldsymbol{r} \mid \boldsymbol{z})\}=\arg \min _{\boldsymbol{r}}\left\{J_{1}(\boldsymbol{r} \mid \boldsymbol{z})\right\} \\
J_{1}(\boldsymbol{r} \mid \boldsymbol{z})=\left\|\boldsymbol{z}-\boldsymbol{H}_{2} \boldsymbol{r}\right\|^{2}+\lambda \sum_{j}\left|r_{j}\right|^{\beta} .
\end{gathered}
$$

When $\boldsymbol{r}$ estimated, we can determine either a binary valued $\boldsymbol{q}$ from it by $q_{j}=1$, if $\left|r_{j}\right|<s$ and $q_{j}=0$ elsewhere, or a real valued $\boldsymbol{q}$ with $q_{j} \in[0,1]$ by $q_{j}=\left|r_{j}\right| / \max \left(\left|r_{j}\right|\right)$ or any other practical solution. This real valued $\boldsymbol{q}$ can be considered as a blurred (or fuzzy) image of borders and edges.

For the second part which is the estimation of $\boldsymbol{x}$ given $\boldsymbol{y}$ and $\widehat{\boldsymbol{q}}$ the following criterion has been used:

$$
\widehat{\boldsymbol{x}}=\arg \max _{\boldsymbol{x}}\{p(\boldsymbol{x} \mid \boldsymbol{y}, \widehat{\boldsymbol{q}})\}=\arg \min _{\boldsymbol{x}}\left\{J_{2}(\boldsymbol{x} \mid \boldsymbol{y} ; \widehat{\boldsymbol{q}})\right\}
$$

with

$$
J_{2}(\boldsymbol{x} \mid \boldsymbol{y}, \widehat{\boldsymbol{q}})=\left\|\boldsymbol{y}-\boldsymbol{H}_{1} \boldsymbol{x}\right\|^{2}+\lambda_{2} \sum_{j}\left(1-q_{j}\right)\left|x_{j+1}-x_{j}\right|^{\beta} .
$$

\section{FUSION OF GEOMETRIC INFORMATION AND RADIOGRAPHIC DATA}

Here, we illustrate an application of the proposed methods for the special case of CT medical imaging where we want to include some geometric information such as partial knowledge of borders of different regions and/or partial knowledge of materials in specified regions of the body in the reconstruction method. The idea of using anatomical information in computed tomography is not new. Many works on the subject has been done before. See for example $[1,11,2]$. Using some partial knowledge of some regions borders can be considered as a special case of the previous example. Actually, in the second step of the proposed method in previous section, we were using the combination of the radiographic data $\boldsymbol{y}$ and $\boldsymbol{q}$ which can be considered as a geometrical (regions borders) data. It has also been used in [1] in medical imaging. Using some partial knowledge about the exact values of pixels in some specified regions has also been used recently [2]. But, combining both region and border informations from anatomic data is new.

In the following, we assume to have sinogram data $\boldsymbol{y}$ and a binary map $\boldsymbol{q}$ containing the borders of some of the regions in the body and $\boldsymbol{s}$ an image containing the attenuation constant values of some of the regions in the body (not forcibly the same regions for which we know the borders) and $\boldsymbol{\mu}$ an image indicating our degree of confidence about the knowledge of values in those regions ( 0 when no knowledge and 1 when high confidence). Based on the MAP Bayesian approach and the discussions in previous sections, 
we propose the following criterion to optimize to find the an image $\widehat{\boldsymbol{x}}$ which will be the result of fusion of these data:

$$
\begin{gathered}
J(\boldsymbol{x} \mid \boldsymbol{y}, \boldsymbol{q}, \boldsymbol{s}, \boldsymbol{\mu})=\left\|\boldsymbol{y}-\boldsymbol{H}_{1} \boldsymbol{x}\right\|^{2}+\lambda_{1} \sum_{j}\left(1-q_{j}\right)\left|x_{j+1}-x_{j}\right|^{\beta_{1}} \\
+\lambda_{2} \sum_{j} \mu_{j}\left|x_{j}-s_{j}\right|^{\beta_{2}}
\end{gathered}
$$

Note that, when the hyperparameters $\lambda_{1}, \lambda_{2}>0$ and $1 \leq \beta_{1}, \beta_{2} \leq$ 2 and the data $\boldsymbol{y}$ and $\boldsymbol{q}, \boldsymbol{s}$ and $\boldsymbol{\mu}$ are given, this criterion is a convex function of $\boldsymbol{x}$. Then, its optimization can be done by any gradient based algorithm. In the following, we show the results obtained by this criterion for the following situations:

- when we do not have any anatomical data $(\boldsymbol{q}=\boldsymbol{\mu}=\mathbf{0})$;

- when we have only the map of borders $\boldsymbol{q}$ but no region data $(\boldsymbol{\mu}=\mathbf{0})$;

- when we have only the map of regions (characterized by both $s$ and $\boldsymbol{\mu})$ but no other borders data $(\boldsymbol{q}=\mathbf{0})$;

- when we have both the borders $\boldsymbol{q}$ and regions maps $(\boldsymbol{s}, \boldsymbol{\mu})$.

In the following, the hyperparameters $\beta_{1}$ and $\beta_{2}$ are fixed either to 2 or to 1.1 and the two regularization parameters $\lambda_{1}$ and $\lambda_{2}$ are adjusted empirically.

The object is a known numerical phantom in CT which has been proposed by Shepp and Logan $[12,13,14]$. This is a $(256 \times$ $256)$ image. The sinogram data is obtained by simulating a fan beam tomography with 64 detectors and 128 angular positions over 0 and 360 degrees for the source. The opening angle for the source is 30.4 degrees. The distance between the source and the center of the object is $600 \mathrm{~mm}$ and the dimensions of the reconstructed image is $(400 \mathrm{~mm} \times 400 \mathrm{~mm})$.

Figure 1 shows the original object and the associated sinogram data. Figure 2 shows the reconstruction results by classical backprojection or filtered back-projection methods used in commercial scanners. As it is seen on this figure, these results are not satisfactory for the data gathering configuration we proposed where we are looking for a high resolution image $(256 \times 256)$ from a sinogram data which has only $(128 \times 64)$ data points $(64$ detectors and 128 source positions uniformly distributed in $0,2 \pi$ ). We also give here two other results obtained by optimizing the criterion

$$
J(\boldsymbol{x} \mid \boldsymbol{y}, \boldsymbol{q}, \boldsymbol{s})=\left\|\boldsymbol{y}-\boldsymbol{H}_{1} \boldsymbol{x}\right\|^{2}+\lambda_{1} \sum_{j}\left(x_{j+1}-x_{j}\right)^{2}
$$

once over $\mathbf{R}^{n}$ and the second over $\mathbf{R}_{+}^{n}$. In both cases, we used a simple gradient algorithm, but in the second case, we imposed the positivity constraint at each iteration. These results are significantly better than the classical back-projection methods thanks to regularization terms, but they need more computations (approximately two times more computations than a simple back-projection in each iteration).

Figure 3 shows the reconstruction results using geometrical data. In this figure, a) and b) show the known region and borders data which are assumed available; c) shows the result when only the region data in a) has been used; d) shows the result when only the borders data in b) has been used; and e) and f) are two results when both region and borders data have been used. These two results have been obtained for two different values of confidence for the regions values (different values of $\lambda_{2}$ which is too low at left but it seems to have good value at right.

Obviously, fusion of more geometrical information results in more accurate results when the geometrical results are exact. Unfortunately, in practical applications, we need a first step of registration to bring the geometrical informations in the same frames of $\mathrm{X}$ ray radiographic data. In previous simulations, we assumed that this has been done, before starting the reconstruction.

In the following, we give some results to show the sensitivity of the results on some errors of registration. Here we simulated the cases where the geometrical atlas data are obtained with some errors on the orientation of some of the known regions ( 5 degree).

Figure 4 shows the results obtained with these errors in the geometrical data with the same conditions which are obtained the results of the figure 3 . In this figure, a) and b) show the errors in regions and borders.
Comparing these results, we see that the degradations due to these errors are not so crucial if the regularization parameters $\lambda_{1}$ and $\lambda_{2}$ are not too high.

There is however the possibilities to reduce these effects, by feedback iterating, i.e. by reestimating these geometrical data from the final reconstructions of the previous results and restarting the data fusion again from these new informations. But, nothing can be given on the convergence of such an iterative procedure.

\section{CONCLUSIONS}

We illustrated the feasibility of a Bayesian estimation approach for a medical computed tomography where we used some anatomical information to obtain better reconstruction results. We used two kind of anatomical information: partial knowledge of values in some regions and partial knowledge of the borders of some other regions. We showed the advantages of using such informations on increasing the quality of reconstructions. We also showed some results to analyze the effects of some errors in anatomic data on the reconstructed results.

\section{REFERENCES}

[1] G. Gindi, M. Lee, A. Rangarajan, and I. G. Zubal, "Bayesian reconstruction of functional images using anatomical information as priors," IEEE Trans. Medical Imaging, vol. MI-12, no. 4, pp. 670-680, 1993.

[2] M. Fiani, J. Idier, and S. Gautier, "Algorithmes ART semiquadratiques pour la reconstruction à partir de radiographies," in Actes du $18^{\mathrm{e}}$ colloque GRETSI, Toulouse, Sept. 2001.

[3] J. Boyd and J. Little, "Complementary data fusion for limited-angle tomography," in Proceeding of Computer Vision and Pattern Recognition 1994, Seattle, WA, USA, 1994, pp. 288-294.

[4] G. Matsopoulos, S. Marshall, and J. Brunt, "Multiresolution morphological fusion of $\mathrm{mr}$ and ct images of the human brain," IEE Proceedings on Vision, Image and Signal Processing, vol. 141 Issue: 3, pp. $137-142,1994$.

[5] J. Boyd, "Limited-angle computed tomography for sandwich structures using data fusion," Journal of Nondestructive Evaluation, vol. 14, no. 2, pp. 61-76, 1995.

[6] S. Gautier, G. Le Besnerais, A. Mohammad-Djafari, and B. Lavayssière, "Fusion de données radiographiques et ultrasonores, en vue d'une applicaion en contrôle non destructif," Clermont-Ferrand, May 1995, Second international workshop on inverse problems in electromagnetism and acoustic.

[7] A. Mohammad-Djafari, "Probabilistic methods for data fusion," in Maximum Entropy and Bayesian Methods, Boise, ID, Aug. 1997.

[8] S. Gautier, G. Le Besnerais, A. Mohammad-Djafari, and B. Lavayssière, Data fusion in the field of non destructive testing, Maximum Entropy and Bayesian Methods. Kluwer Academic Publ., Santa Fe, NM, K. Hanson edition, 1995.

[9] S. Gautier, Fusion de données gammagraphiques et ultrasonores. Application au contrôle non destructif, Phd thesis, Université de Paris-Sud, Orsay, Dec. 1996.

[10] S. Gautier, G. Le Besnerais, A. Mohammad-Djafari, and B. Lavayssière, "Vers la fusion de données gammagraphiques et ultrasonores," in Actes du $15^{\mathrm{e}}$ colloque GRETSI, Juan-les-Pins, Sept. 1995, pp. 869-872.

[11] S. Gautier, J. Idier, A. Mohammad-Djafari, and B. Lavayssière, "X-ray and ultrasound data fusion," in Proc. IEEE ICIP, Chicago, IL, Oct. 1998, pp. 366-369.

[12] B. F. Logan and L. A. Shepp, "Optimal reconstruction of a function from its projections," Duke Math. J., vol. 42, pp. 645-659, 1975. 
[13] L. A. Shepp and Y. Vardi, "Maximum likelihood reconstruction for emission tomography," IEEE Trans. Medical Imaging, vol. MI-1, pp. 113-122, 1982.

[14] G. T. Herman, Image reconstruction from projections. The fundamentals of computerized tomography, Academic Press, New York, 1980.
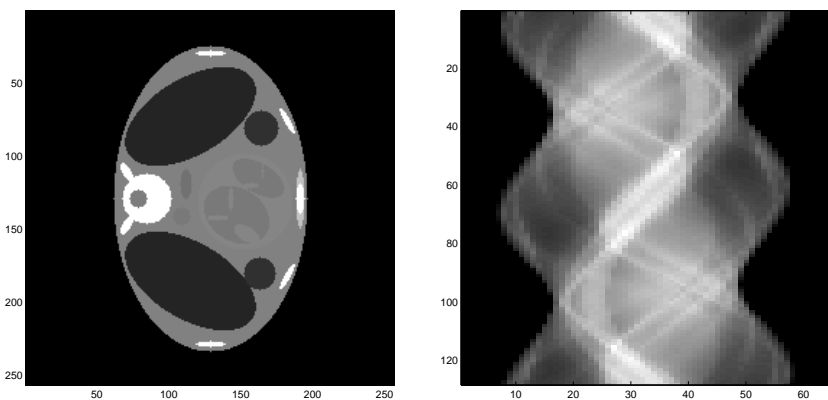

Fig. 1. Original object (left) and its sinogram data (right). The geometry is a fan beam CT, the object is $(256 \times 256)$ and the sinogram data is $(128 \times 64)$.

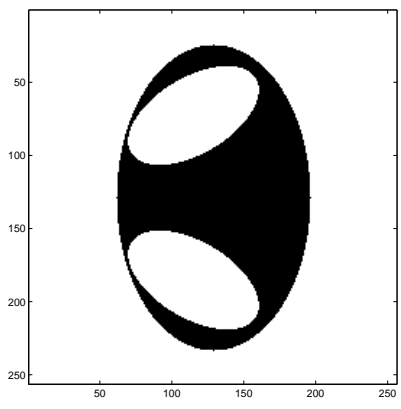

a)

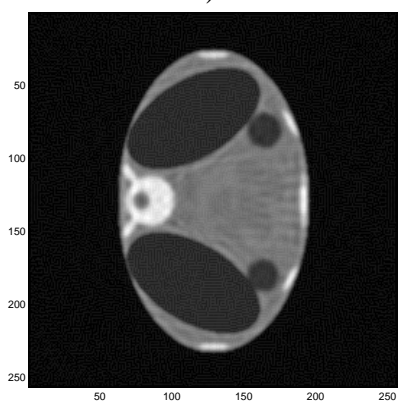

c)

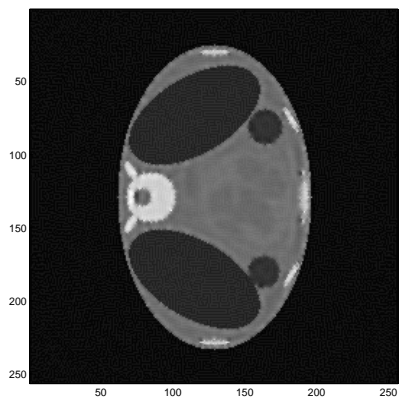

e)

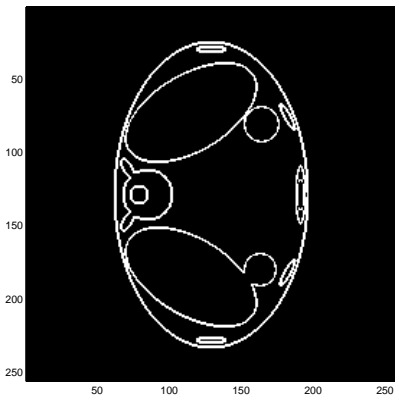

b)

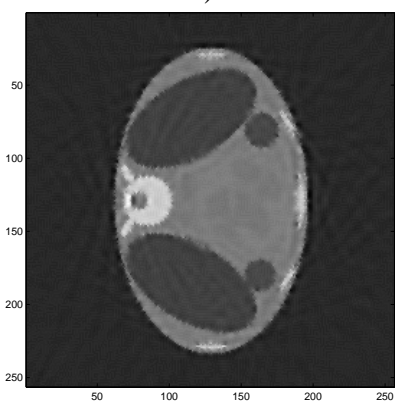

d)

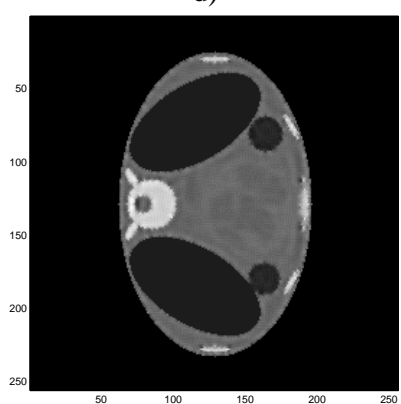

f)
Fig. 3. Reconstructions by data fusion (exact geometrical data): a) known regions data, b) known borders data;

c) Results using a), d) Results using b);

e) and f) Results using both a) and b) for two different values of confidence for the regions values (different values of $\lambda_{2}$ which is too low at left but it seems to have good value at right.

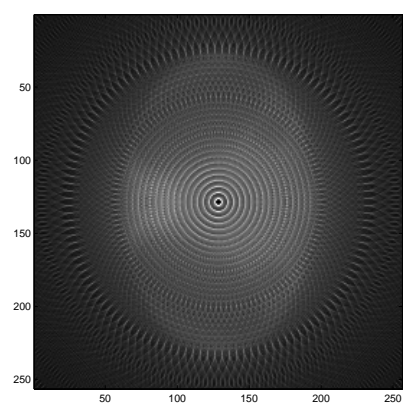

a)

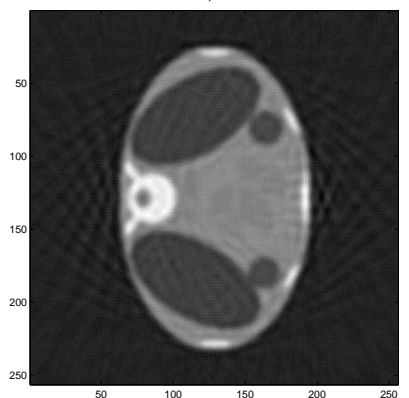

c)

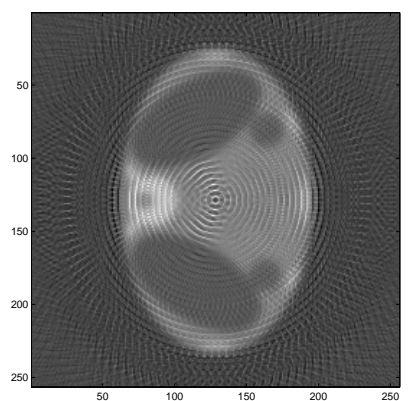

b)

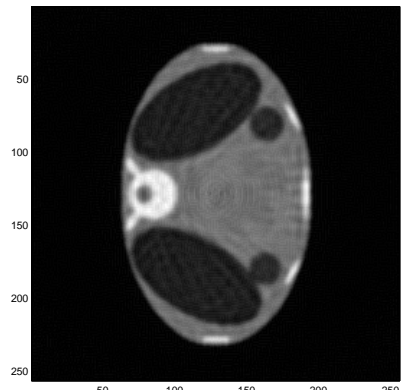

d)
Fig. 2. Reconstructions by classical methods: a) back-projection b) filtered back-projection c) quadratic regularization and d) quadratic regularization with positivity constraint.

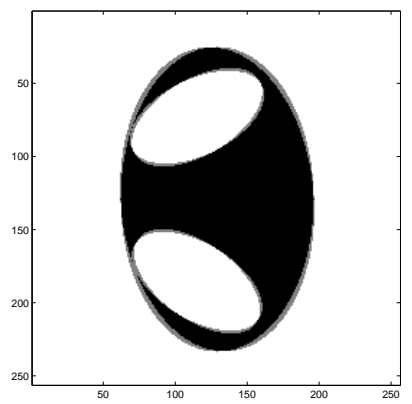

a)

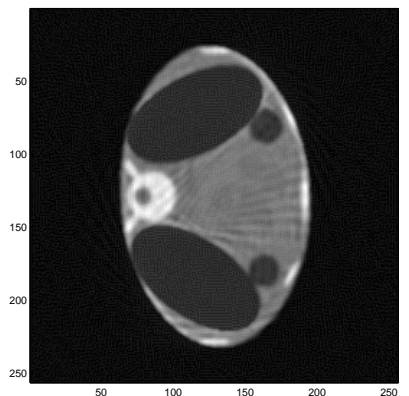

c)

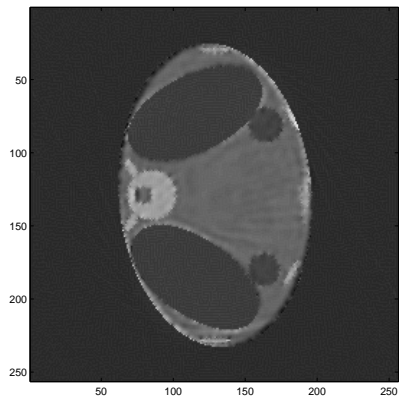

e)

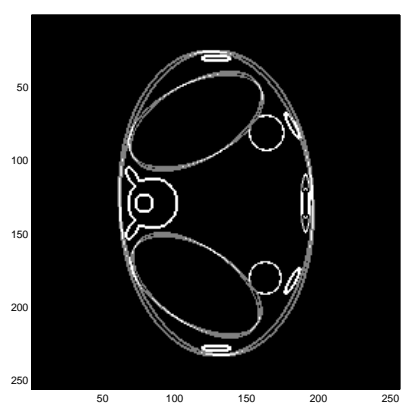

b)

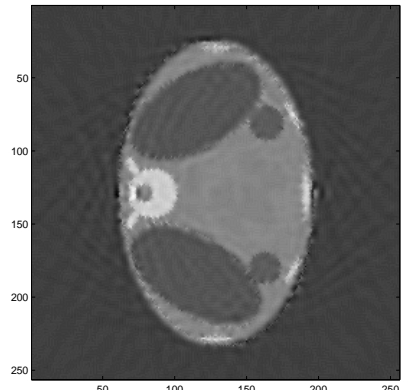

d)

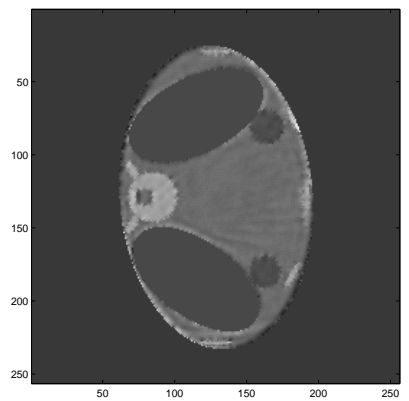

f)
Fig. 4. Reconstructions by data fusion with errors in the geometrical data: a) known regions with errors, b) known borders with errors; c) result using a), d) result using b); e) and f) results using both a) and b) for two different values of confidence for the regions values. These results are to be compared with those of Fig. 3 which were obtained with exact geometrical data. 